\title{
Site-scale variability of calling activity of insectivorous bats: implications for passive acoustic monitoring
}

\author{
Alexander Bruckner ${ }^{1}$ and Lisa Greis ${ }^{2}$ \\ ${ }^{1}$ University of Natural Resources and Life Sciences Vienna \\ ${ }^{2}$ Affiliation not available
}

February 15, 2021

\begin{abstract}
Detectors for the passive acoustic monitoring (PAM) of bats have become invaluable research tools, especially for surveys, monitoring programs and environmental impact assessments. However, little is known about the small-scale (within-site) variability of PAM recordings and especially about the influence of detector identity and distance, and of microphone orientation on the statistical confidence of activity estimates and species detection probabilities. We recorded vocalizations in a homogeneous meadow with no trees, bushes or tall ground vegetation. Eight detector pairs were arranged in an octagon, the two detectors of a pair facing in opposite directions. The call sequences of eight species were analyzed. The deviations of individual detectors from the overall mean were generally small, but large outliers occurred both at the file (temporal resolution: five seconds) and the night (resolution: one night) scale. All devices detected the main temporal patterns of calling activity in the study period, but three devices deviated systematically from the others and the sensitivity of two devices deteriorated over time. Detector orientation and distance were significant, yet small, sources of variability. The probability of detecting the presence of species correlated with species' activity and ranged on average from $100 \%$ for bats in total to only $18.8 \%$ for the least active Myotis myotis. The sample sizes necessary to achieve $90 \%$ statistical confidence of activity estimates ranged from 7 to 16 detectors and from 5 to 12 nights, depending on taxon. Increasing the number of nights resulted in much higher confidence than increasing the number of detectors. We recommend PAM studies of bats to frequently calibrate detector sensitivity; deploying at least three detectors per study site; sampling longer periods instead of deploying more detectors; randomly assigning and swapping detectors among sites, treatments, strata, etc.; and statistically scrutinizing the sample data, especially for outliers.
\end{abstract}

\section{Hosted file}

MS_EcolEvol.pdf available at https://authorea.com/users/395879/articles/508974-site-scalevariability-of-calling-activity-of-insectivorous-bats-implications-for-passive-acousticmonitoring 


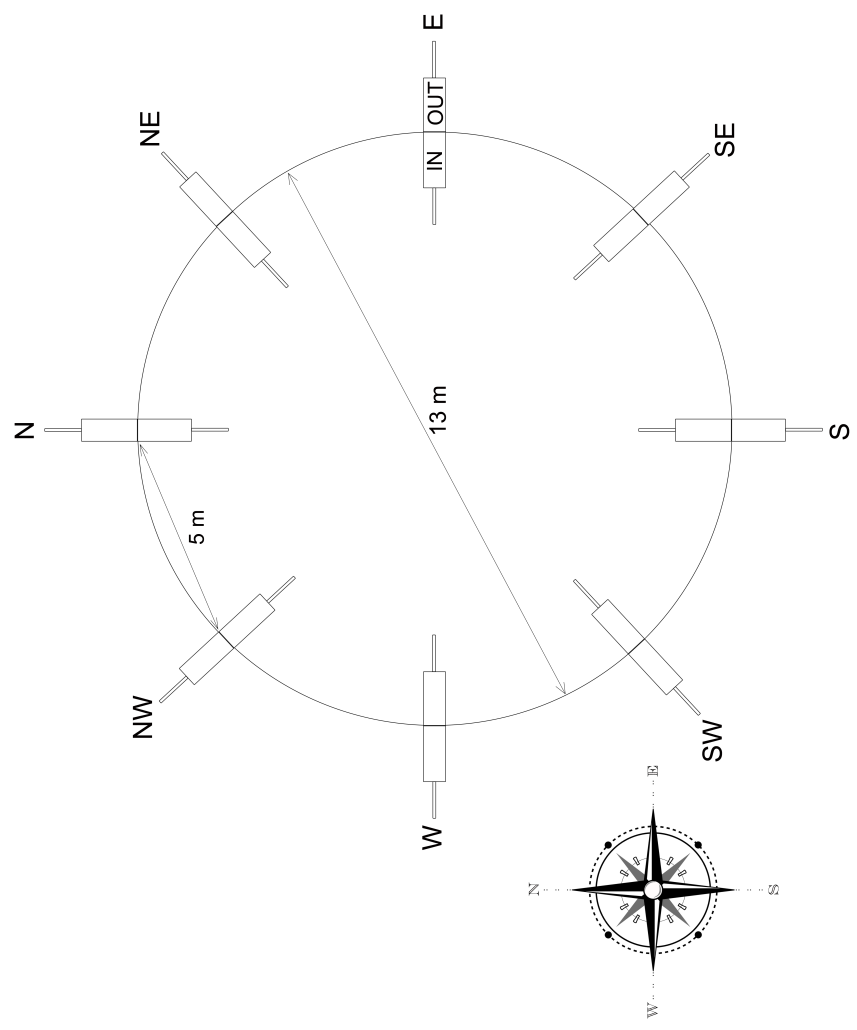



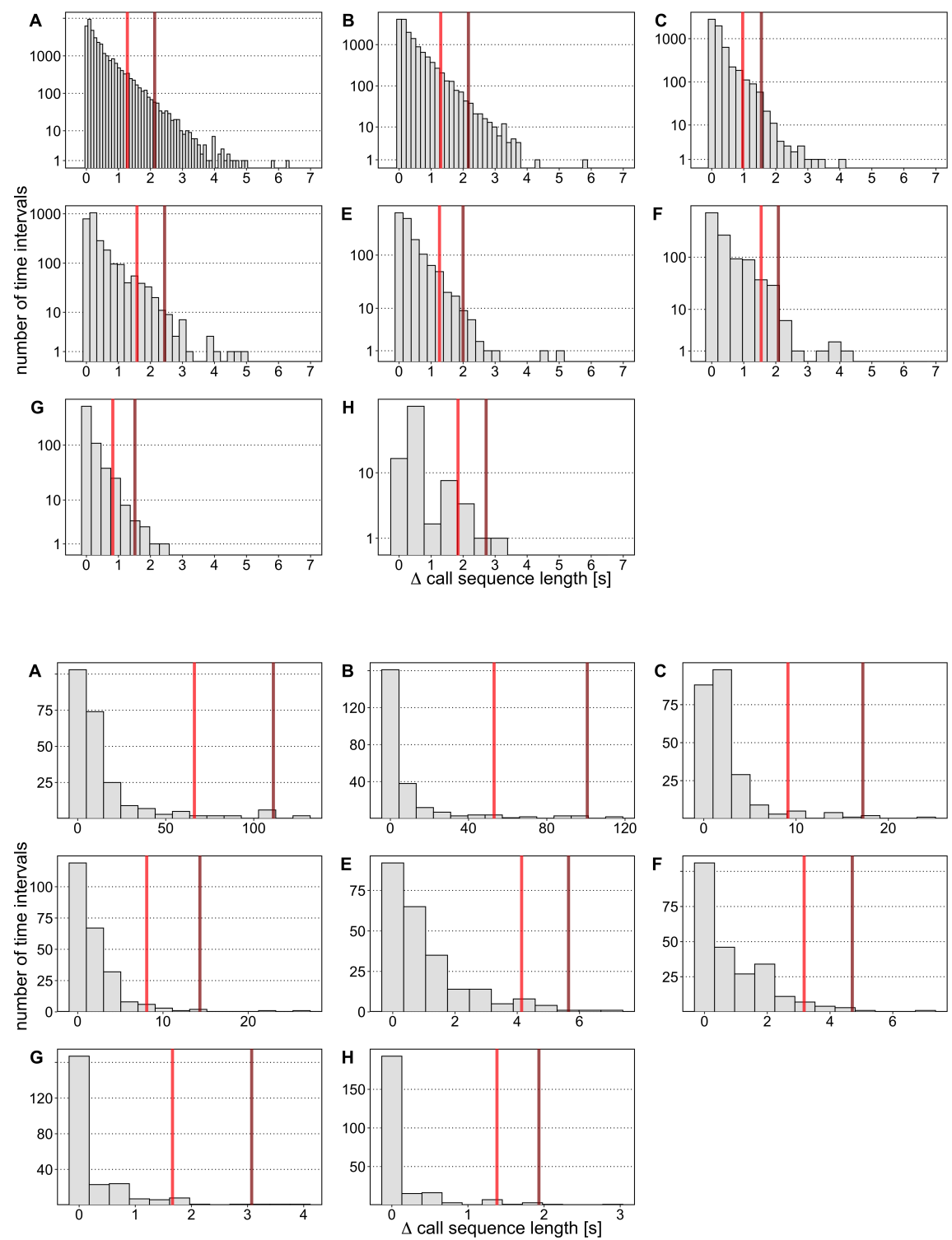

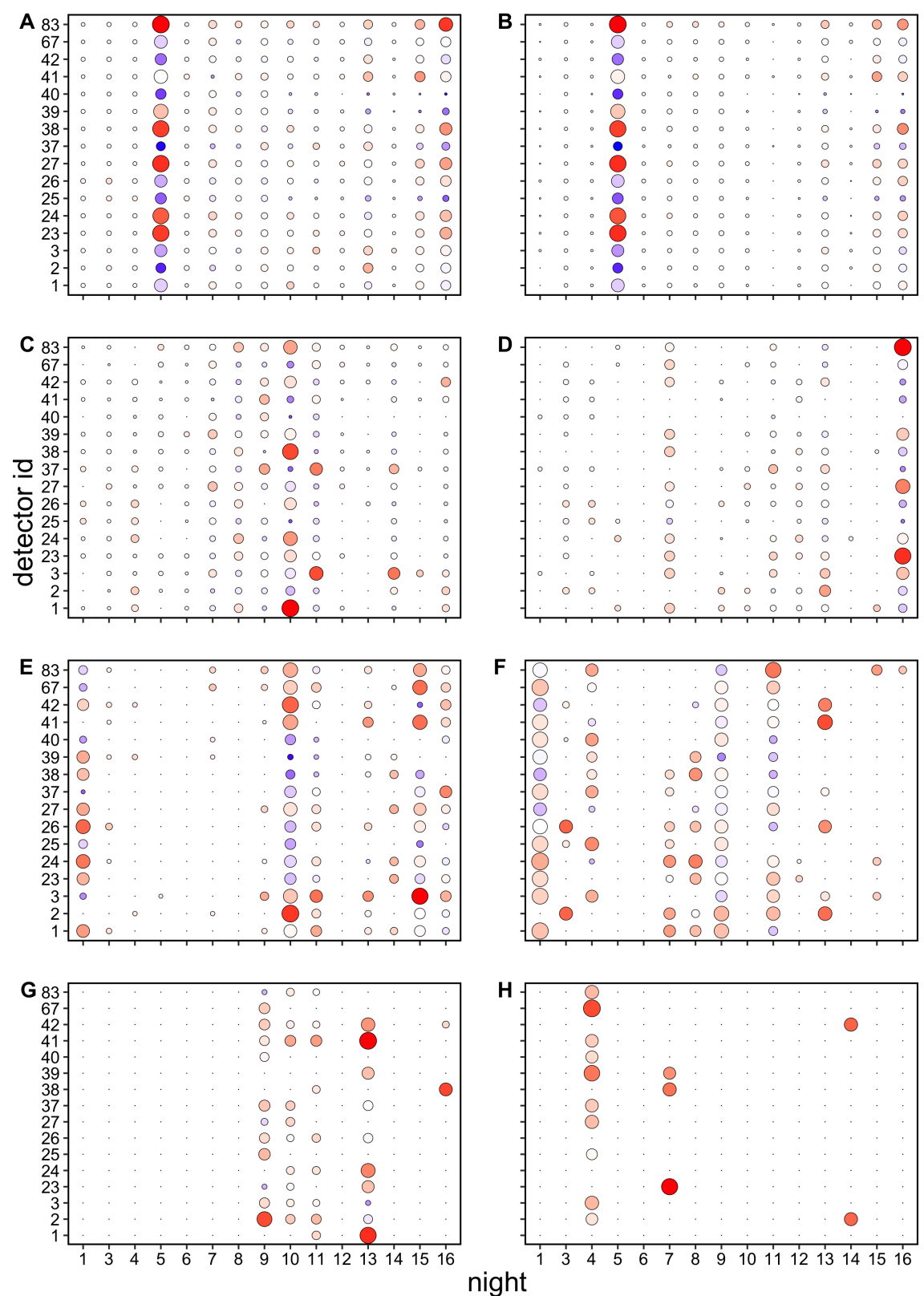

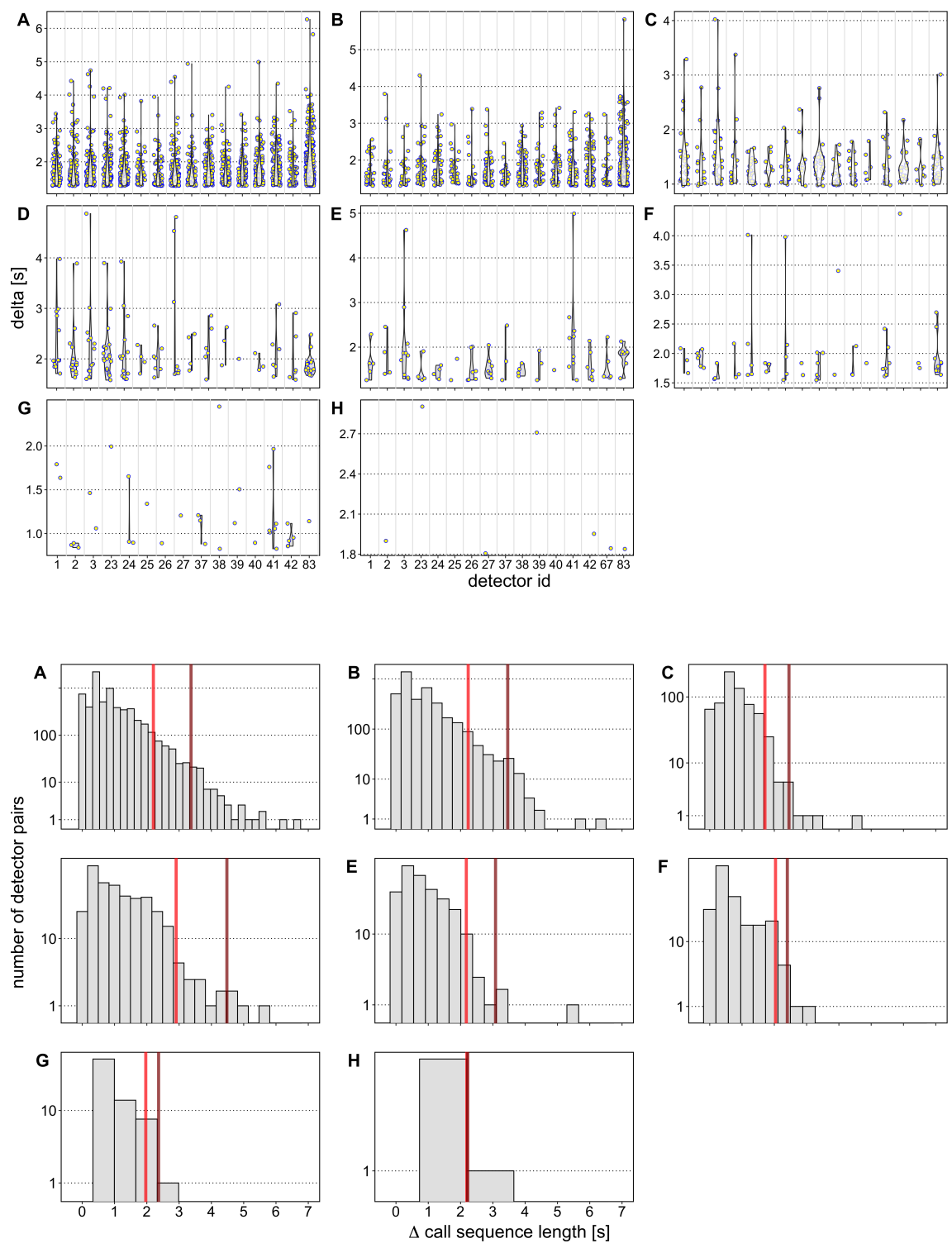

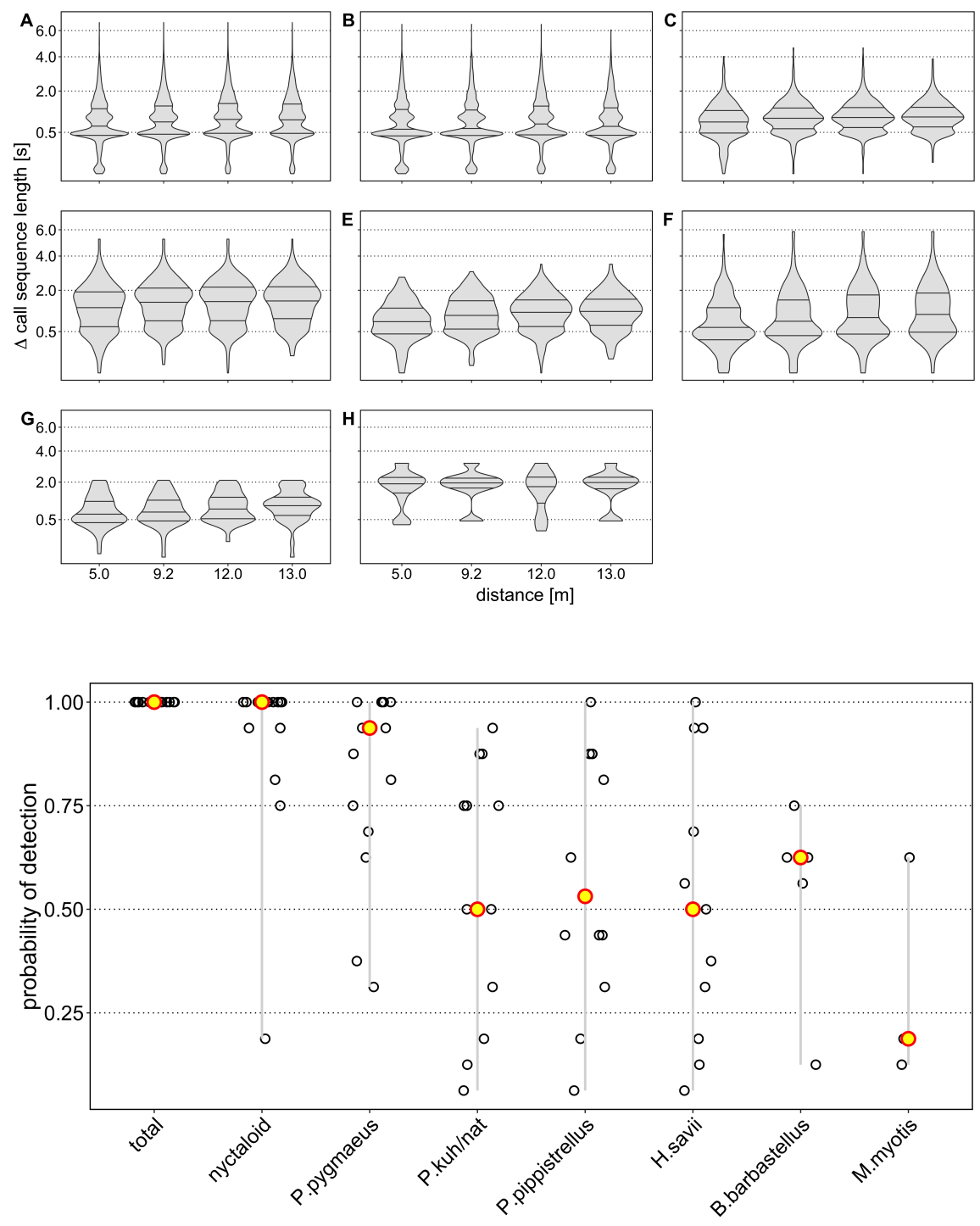

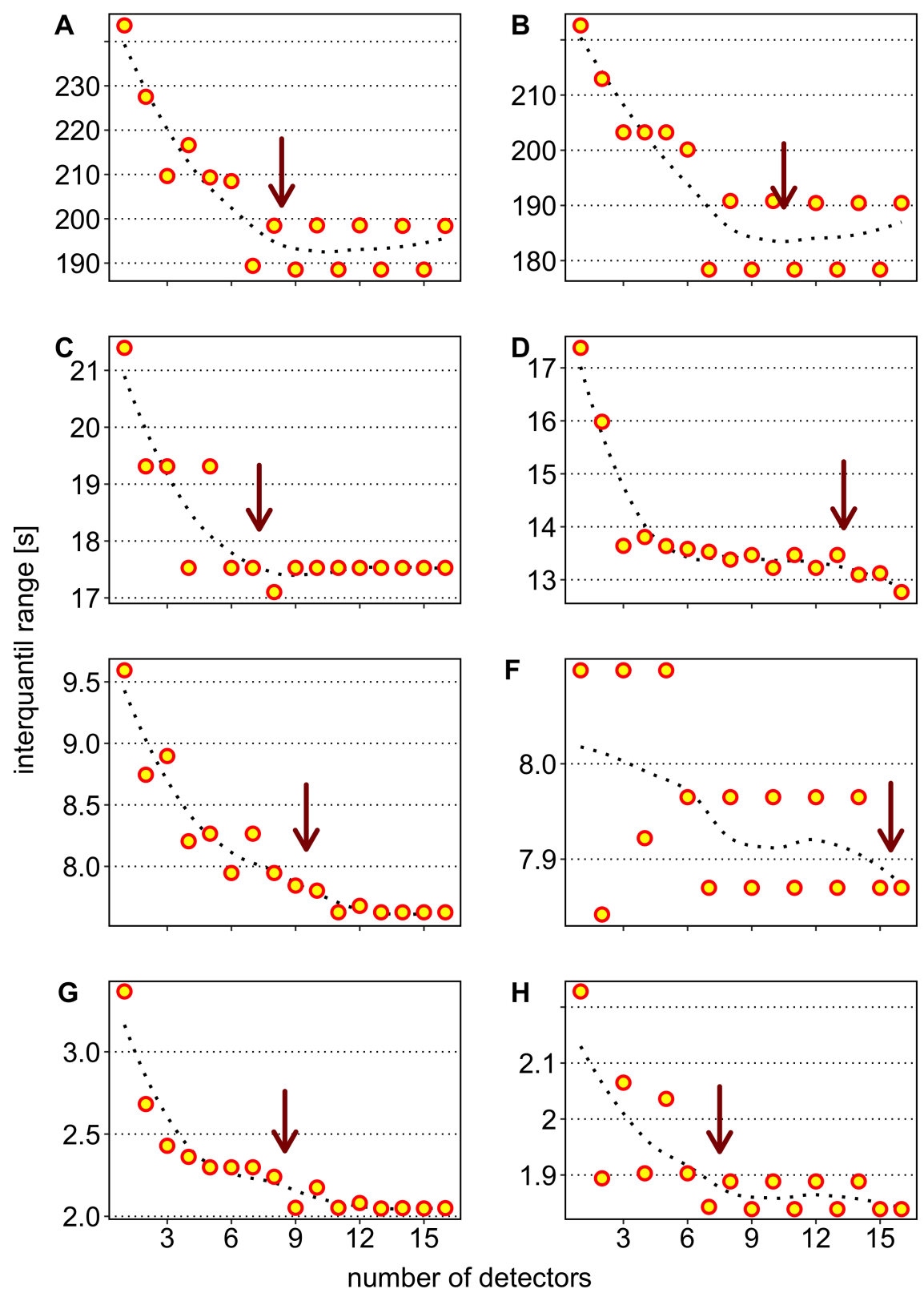

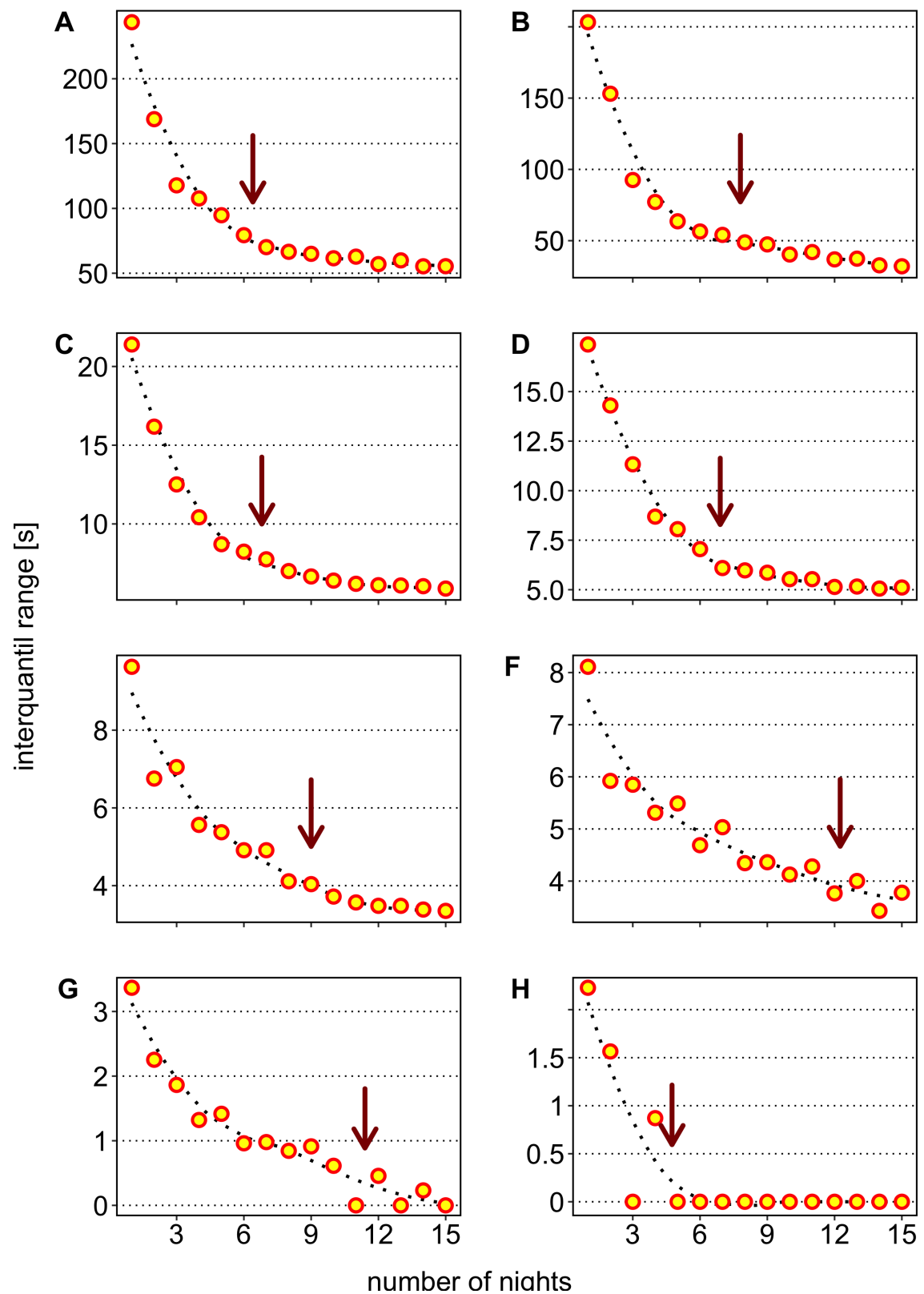